\title{
Las PyMEs en Santa Cruz, Análisis, Distribución e Innovación Vinculada al uso de las TICS
}

\section{SMEs in Santa Cruz, Analysis, Distribution and Innovation Linked to the use of Information and Communication Technologies.}

\author{
Alejandro Aroca Bavich \\ UTN. Facultad Regional Santa Cruz. Grupo GIGEI. \\ arocabavich@gmail.com
}

\section{Daniel Martínez Llaneza}

UTN. Facultad Regional Santa Cruz. Grupo GIGEI.

\section{Iván Ossandon}

UTN. Facultad Regional Santa Cruz. Grupo GIGEI.

\section{Rafael García}

UTN. Facultad Regional Santa Cruz. Grupo GIGEI.

\section{Resumen}

El proyecto de investigación asociado a esta publicación pretende el estudio y acercamiento a las Pymes radicadas en la región con el fin de determinar los rasgos sobresalientes que estas tienen en materia de gerenciamiento. Se buscó indagar sobre las Pymes para determinar concretamente si realizan Planeamiento Estratégico, si las politicas de Recurso Humanos se vinculan a dicho Planeamiento y si desde estas empresas se incorpora innovación tecnológica y de qué modo incorpora el avance tecnológico en su función de producción. A tales fines se llevó adelante una campaña de relevamiento mediante encuestas que permitió estudiar e inferir el comportamiento de las organizaciones empresariales al respecto de varios de estos aspectos, la presente publicación logra caracterizar las pymes en conceptos generales y da evidencia del avance e incorporación de las Tecnologías 
de Información y Comunicación en las PyMEs, explicando los principales mecanismos de innovación en esta materia.

Palabras claves: PyMEs, Santa Cruz, Planeación Estratégica, Recursos Humanos, TIC

\begin{abstract}
The research project asociated to this publication aims to study and approach the University to the SMEs located on the region in order to determine the outstanding features in matter of Management. The project searched to investigate SMEs and determine specifically if it do Strategic Planning, if the Human Resource policies are linked to the planification and if these companies are incorporating tecnological innovations and how it incorporates technological advancement in the production function. To acomplish this objetive it has been made a survey campaign that allow the study and inferring the behavior of the organizations in respect of several of these aspects, this publication, has achieved characterize the SMEs in general concepts and evidence the progress and the incorporation of Information and Communication Technologies in SMEs, explaining the main innovation mechanisms in this area.
\end{abstract}

Keywords: SMEs, Santa Cruz, Strategic Planification, Human Resources, ICT 


\section{INTRODUCCIÓN}

El proyecto vinculado a esta publicación, lleva como meta el estudio de las Pequeñas y Medianas empresas radicadas en la Provincia de Santa Cruz, con el fin de determinar la forma en que estas administran y vinculan el Planeamiento Estratégico, la Innovación y sus Políticas de Recursos Humanos. Se buscó establecer si el empresario PyME identifica que existe este vínculo entre ellas y por tanto busca armonizarlas y condiciona las políticas empresariales adoptadas en función de los factores expuestos.

Este trabajo se enfocó puntualmente en establecer los resultados parciales de la investigación, en función de cómo las PyMEs regionales han adoptado las nuevas Tecnologías de Información y Comunicación como fuente de Innovación Tecnológica para potenciar sus negocios y del uso de otras herramientas que le permitan incorporar innovación en su organización y optimizar el gerenciamiento de los Recursos Humanos.

En este análisis del vínculo de la Innovación y las políticas de Recursos Humanos tanto en corto y el largo plazo, se buscó evaluar el grado de absorción de la innovación tecnológica o el modo en que se incorporó el avance tecnológico en su función de producción.

Se buscó esclarecer las conductas empresarias habituales, y el grado de difusión que estas tienen, dentro del sector privado de la provincia de Santa Cruz, como también fuera de ella.

El objeto principal del trabajo radica en identificar si las Pymes de Santa Cruz, en su ámbito directivo, generan planificación estratégica y diseñan políticas vinculadas para concretar estas estrategias, como así también el modo en que el gerenciamiento administra estrategias de recursos humanos y las TICS para competir empresarialmente.

\section{MARCO TEÓRICO}

\section{La Gestión de los Recursos Humanos}

Los recursos humanos de la empresa, deben constituirse como una ventaja competitiva que posea la propia organización.

Por ello, en este concepto radica que la Gestión Estratégica de Recursos Humanos (GERH) considere al personal que forma parte de la organización, como una Inversión, ya que se trata de un recurso decisivo para la competitividad de cada empresa u organización, tenga esta fines de lucro o no. 
La estrategia de la empresa debe alinearse con los recursos humanos de manera tal que sea procedente una gestión coherente y centrada en un desarrollo a largo plazo (Bohlander, G., \& Snell, S. 2006).

El enfoque sistémico, multidisciplinario, proactivo y de proceso resultan esenciales para la GERH, por ello se dice que el sistema de gestión de recursos humanos es más que la suma de las partes, lo que potencia su rol de sistema y su sinergia dentro de la organización, así el carácter sistémico resulta esencial para la funcionalidad del proceso de GERH (Cuesta Santos, A. 2005. Cuesta, Armando. 2002)

Las prácticas de Recursos humanos pueden ser herramientas poderosas para mejorar la efectividad de las organizaciones que compiten sobre la base del conocimiento (Ferris, G. R., Hall, A. T., Royle, M. T., \& Martocchio, J. J. 2004).

Los diferentes aportes, dan cuenta del fuerte interés en la rivalidad de mercado, el posicionamiento a largo plazo, la dirección de acción a futuro, planteo de objetivos amplios, políticas principales y asignación de recursos. Cada uno de estos términos implica considerar la gestión de los recursos humanos como un factor central para el desarrollo de la organización.

Resulta claro que el no considerar apropiadamente este factor, limitara el desarrollo de las otras áreas de la organización, por ello las nuevas concepciones de manejo empresarial los consideran ampliamente.

\section{La dirección estratégica}

La dirección estratégica, referencia a todas las materias que permiten estudiar los factores de éxito o fracaso de las organizaciones (Llanes, W. 2004).

Definiciones más descriptivas de otros autores (Porter 1982, Yoguel 1996 Harper 1992, Llanes 2004), logran representar a la dirección estratégica de una organización, como la estructura teórica utilizada para la reflexión de las grandes opciones de la empresa que sugiere la práctica de una nueva cultura y actitud, vinculando lo estratégico a lo operativo de forma sistemática y coherente.

Este conjunto de conceptos además de estar fuertemente orientados a la operatividad, supusieron un nuevo modelo, mayoritariamente por la aceptación del carácter sistemático que se le otorga.

La tecnología ha generado cambios en los paradigmas de creación de valor, se pueden observar cadenas de valor que se estructuran de forma diferente a lo planteado por el antiguo paradigma industrial, puede observarse que en la actualidad el valor de un gran conjunto de bienes está asociado en mayor cuantía con la Investigación, el Desarrollo y la innovación (I+D+i) que estos tengan incorporados.

La inversión que lleva a cabo una empresa y su posicionamiento a largo plazo tiene que ver con la estrategia que esa empresa lleva adelante, así tomando a Porter M. (1982) se indica el rol esencial de la estrategia competitiva consiste esta en desarrollar una amplia fórmula de cómo la empresa va a competir, cuáles deben ser sus objetivos y que políticas serán necesarias para alcanzar tales objetivos en el largo plazo.

Otros autores hacen referencia a la estrategia empresarial como la dialéctica de la empresa con su entorno (Pena 2004). 
Dando lugar a una visión de largo plazo se dice que la estrategia es un plano, es decir, una dirección de acción para el futuro, un camino para ir de un punto a otro del desarrollo en la organización. (Mintzberg, H. 2003)

Rogelio Gárciga (1999) destaca los aspectos más relevante de la estrategia al decir que la esta es el producto de un conjunto de acciones lógicas y creativas aplicables que conducen a la formulación de objetivos amplios, de políticas principales y de asignación de recursos para lograr las metas trascendentales de una organización, en la búsqueda de una mayor posición competitiva y una respuesta más coherente entre el entorno actual y futuro.

\section{Los nuevos paradigmas}

Los cambios de paradigma están fuertemente relacionados con la formas de articulación del factor trabajo con los bienes y otros factores, mayoritariamente con una fuerte intervención de las relaciones virtuales y asincrónico, apartándose de cierta manera del contacto presencial habitual.

Una de las ventajas competitivas de una organización radica en sus recursos humanos, por lo que es de esperar que la calificación operativa de estos sea cada vez más determinante.

Muchas actividades económicas eligen una determinada localización dependiendo de la disponibilidad de recursos humanos.

Por tanto resulta de sumo interés mantener ese recurso motivado y compenetrado con el objetivo de la organización.

La actual GERH requiere un enfoque sistémico, multidisciplinario, participativo, proactivo y de proceso. Uno de los propósitos de la GERH es el aumento de la productividad del trabajo así como de la satisfacción laboral, vinculada a las condiciones de trabajo. (Cuesta Santos 2005).

El objetivo de preservación del medio ambiente, también constituye un objetivo sustentable de la GERH.

Los enfoques de maximización de la productividad laboral, no son aceptables de acuerdo a autores recientes sin ser asociados a la sustentabilidad. Por ello se habla de máximo rendimiento sostenible cuando se analiza la calidad de vida laboral (Sánchez Dayana Lorena 2003), otros autores hablan de productividad sostenible o sistemas de trabajo sostenibles pero todos apuntan a que los aumentos de productividad deben sustentarse en un puesto de trabajo mucho más adaptado a quien lo ejerce.

\section{Las TICs como herramientas de Innovación y transformación Productiva}

Los procesos de transformación productiva, el desarrollo de proceso de creación de valor, diversificación de exportaciones y el desarrollo de nuevos productos, y servicios son potenciados en gran medida por el uso de las TICs al ser estas consideradas como elementos dinamizadores de la Innovación, el aprendizaje y gestión 
del conocimiento (Hernandez Valencia R. A., et Al 2009)

En base a lo expresado por Castells (2005), las TICs toman un rol protagonista en la transformación productiva de la sociedad, dado que propicia las acciones de conocimiento

Villaseca afirma que las TICs generan un nuevo paradigma tecnológico y alteran la dinámica de la revolución industrial, tendiendo a la transformación de la realidad social y económica a una realidad nueva basada en el uso y apropiación del conocimiento. (Villaseca Et al 2003)

Peirano manifiesta que las PyMes Argentinas abordaron el uso de las TICs en tres niveles, el primero de ellos para abaratar y agilizar sus costos operativos, el segundo para mejorar el proceso de toma de decisiones además de lo planteado para el primero y el tercero para potenciar y mejorar las actividades de innovación además de los usos antes mencionados. (Peirano et $\mathrm{Al} 2006$ )

\section{Limitaciones al análisis de la PyMes santacruceñas}

El estudio de los procesos de desarrollo empresario regional con inserción socio económico, debe considerar diferentes dimensiones de análisis:

a) Las condiciones y posibilidades jurisdiccionales y económicas de Santa Cruz en cuanto a los recursos cuya explotación hoy se permite

b) Las posibilidades estructurales y sistémicas de la economía nacional y su vínculo con el sistema local y global

c) El marco y el funcionamiento institucional y regulatorio de los mercados y las intervenciones públicas

d) La composición y comportamiento del sector privado.

e) Los recursos y condiciones socio-culturales en los espacios productivos provinciales y locales

f) Los proyectos y modelos dominantes de crecimiento económico.

Los temas de estudio de este Proyecto de Investigación son pertinentes al marco teórico-metodológico que ofrece la teoría de los sistemas complejos.

Para el análisis de cada empresa seleccionada y posterior producción de los diagnósticos correspondientes, se trabajará con información cualitativa y cuantitativa para los diferentes niveles de análisis considerados. En tal sentido se establecieron técnicas de recolección de datos y de procesamiento de la información recopilada, de acuerdo a sus particularidades

\section{METODOLOGÍA Y RECOLECCIÓN DE INFORMACIÓN SOBRE EL TEJIDO PYMES}

En primera instancia para lograr un correcto desarrollo que resulte representativo de la región, fue necesario estudiar la distribución de PyMEs en la provincia de Santa Cruz, de modo tal que se pudo establecer un muestreo adecuado en relación 
con la actividad.

Se analizó la distribución geográfica poblacional de la provincia como punto de partida para identificar las localidades de mayor interés, obsérvese la Figura 1

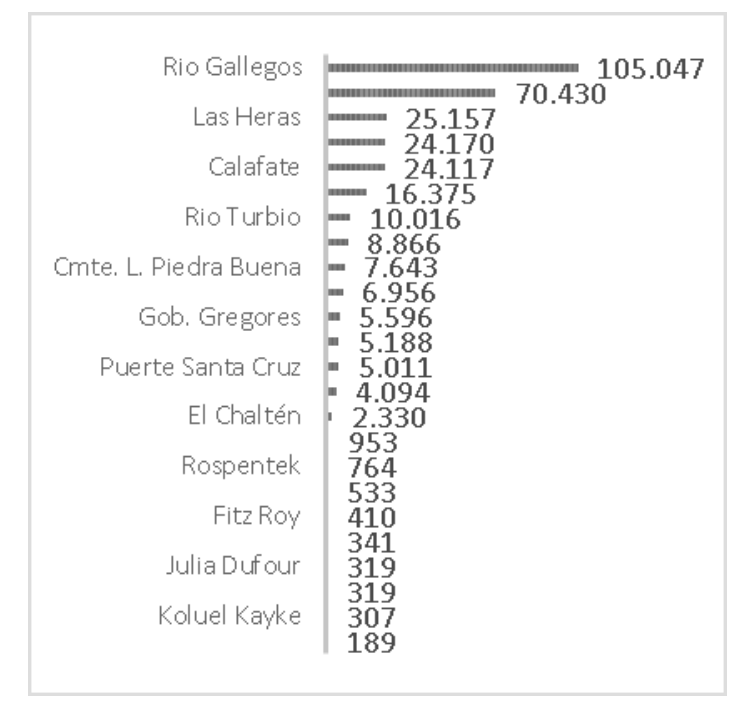

Figura 1. Distribución Poblacional en la Provincia de Santa Cruz - (INDEC 2015)

Dichos valores fueron segmentados y representados en la Figura 2 por tres zonas que se consideraron representativas para la distribución, Zona Norte, Zona Centro y Zona Sur.

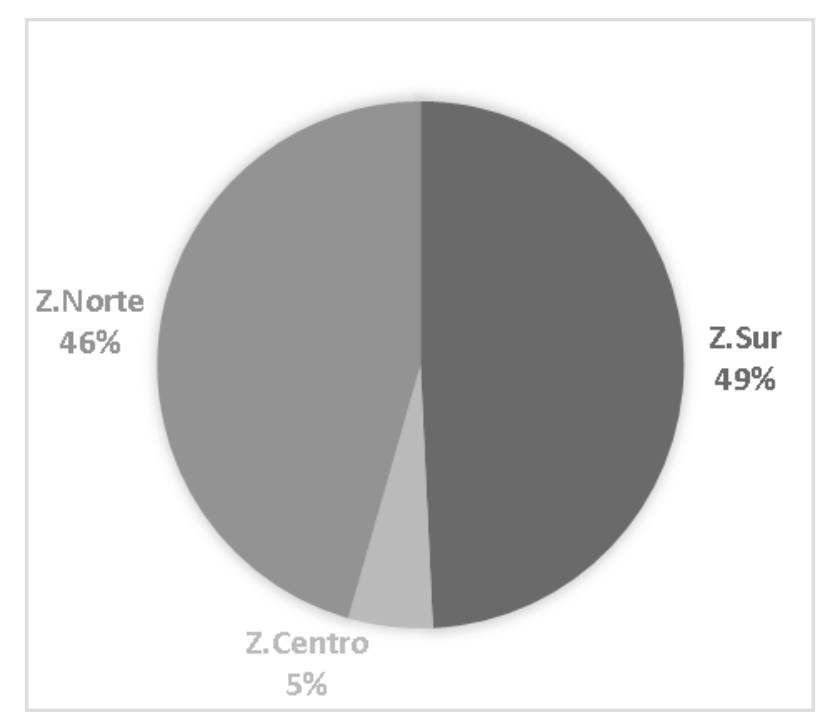

Figura 2. Distribución poblacional en la Provincia de Santa Cruz agrupada en Zonas. 
En lo referente a la Zona Centro de la provincia fueron consideradas las Localidades de Puerto San Julián, El Chalten y Gobernador Gregores. Siendo ellas además adoptadas como separación geográfica para las localidades que fueron comprendidas dentro de cada categoría.

Para en análisis particular en cada localidad, se confeccionaron padrones de empresas PyMEs, y se adoptaron como criterios de exclusión aquellas que tenían una cantidad menor de 5 empleados o mayor de 100 empleados.

Se confeccionaron padrones de empresas en función de la información que pudo ser recabada en cada uno de los diversos Organismos consultados.

Ello implico entes Nacionales, provinciales y Locales relacionados con la actividad, tanto organizaciones estatales como privadas.

Resulto un padrón total de 413 empresas las cuales fueron distribuidas por localidad y posteriormente seleccionadas de forma aleatoria en cada uno de los grupos, cuidando de lograr una muestra lo más heterogénea posible. Se refinaron estos padrones para evitar ponderar demasiadas empresas de un mismo rubro.

Para la recolección de datos se confecciono una encuesta que concluyo con 31 preguntas.

Estas preguntas buscar revelar información significativa con diversos mecanismos basados en la premisa de plantear preguntas simplificadas que puedan correlacionarse con los criterios de interés mencionados.

Se buscó evitar la dificultad de solicitar al empresario respuestas sobre conceptos específicos asociados de manera directa con el marco teórico.

Se diseñaron las preguntas de modo reducir al mínimo la variación por interpretación, para minimizar el sesgo en los datos obtenidos.

Una vez que fueron realizados todos estos pasos, se generó un escrito de presentación del grupo de trabajo y encuesta para los empresarios.

Esta incluyo además las referencias que permitían al encuestado conocer los beneficios y el alcance tanto de la Ley 17.622 de Secreto estadístico como de la ley 24.766 sobre confidencialidad.

Se diseñó la encuesta de forma tal que fuese anónima y los datos fueron procesados de forma que no pueden relacionarse con los resultados publicados.

Todo ello con la premisa de que el empresario no tuviese que revelar información económica que pudiesen incomodarlo, de modo tal que en la mayoría de los casos se utilizaron segmentos representativos para analizar la información.

En posteriores líneas de trabajo que surgen dentro de este proyecto se prevé realizar entrevistas con grupos representativos de estas empresas, para enriquecer la información y lograr obtener un conocimiento profundo sobre la visión del empresario en su entorno.

En primera instancia las encuestas se planificaron y ejecutaron por intermedio de internet y sus herramientas, lo que permitió realizar esta recolección en línea del $20 \%$ del padrón propuesto.

Dadas las dificultades presentadas durante el relevamiento, se optó 
por aumentar el número de muestras obtenidas en base a la recolección de campo.

Se realizaron visitas a cada una de las localidades de la provincia para efectuar la encuesta en forma presencial.

Para fortalecer esta instancia se propendió al contacto institucional a través de los organismos de representación como cámaras de comercio, cámaras sectoriales y similares que nuclean la actividad de las PyMEs.

Como resultado final se logró relevar 65 PyMEs en la provincia, según la distribución observada en la Figura 3.

Se decidió ponderar la muestra inicial reforzando levemente la zona centro de la provincia, dada la fuerte influencia del sector Minero y Turístico en estas localidades.

Resultando de esta manera una interpretación más realista del agregado económico que incorporan estas actividades a la matriz productiva de la provincia.

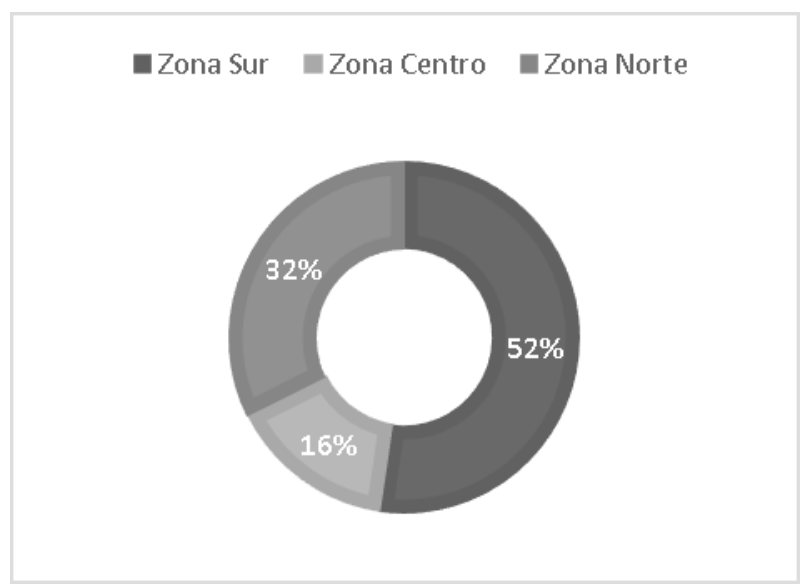

Figura 3. Distribución geográfica de Pymes lograda.

\section{LOS RESULTADOS. EL ANÁLISIS DE LOS DATOS}

Para analizar de manera concreta la forma en que las organizaciones se han adaptado dentro de las nuevas corrientes de innovación y las TICs, se consideró necesario realizar un estudio sobre la edad promedio de las organizaciones PyMEs que fueron encuestadas.

Para ello fue planteada una pregunta enfocada sobre la cantidad de años que poseían la actual conformación societaria e indagaciones sobre las conformaciones anteriores y motivos de cambio. Con ello se pudo construir información adecuada para estudiar el periodo de actividad de cada empresa. 


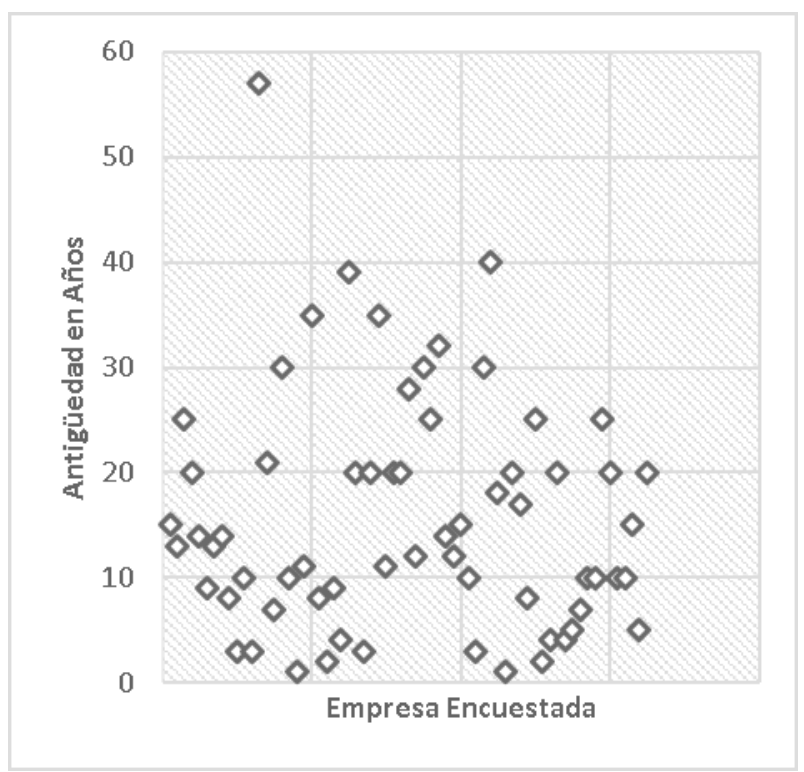

Figura 4. Dispersión de Antigüedad de las organizaciones relevadas.

Analizando la Figura 4 se observa que la antigüedad promedio registradas de las organizaciones resulto en 16 años, lo que indica claramente que la mayoría de estas organizaciones dada su longevidad, tuvieron que adaptarse a los cambios tecnológicos e informáticos.

Resulto importante evaluar fue la cantidad de empleados que participan en estas pymes, con especial énfasis en eliminar aquellos que estuvieron fuera de los criterios de exclusión mencionados.

Para ello se decidió agrupar las categorías y cantidad de trabajadores en 5 segmentos, los cuales son detallados en la Figura 5 permitiendo un análisis de la cantidad de mano de obra como así también una medida indirecta del tamaño promedio de las PyMEs involucradas.

Del análisis se desprende que gran parte de las PyMES muestreadas emplean una cantidad alta de empleados.

Se asocia este comportamiento a las empresas relacionadas al turismo en mayor medida.

La distribución de empleados permite destacar un salto de escala importante entre los estratos segundo y tercero, con respecto al quinto.

Puede asociarse este comportamiento a los costos de escala que posiblemente obliguen a las organizaciones a crecer deliberadamente en la cantidad de empleados contratados, ejecutando un salto apreciable y por tanto costoso. 


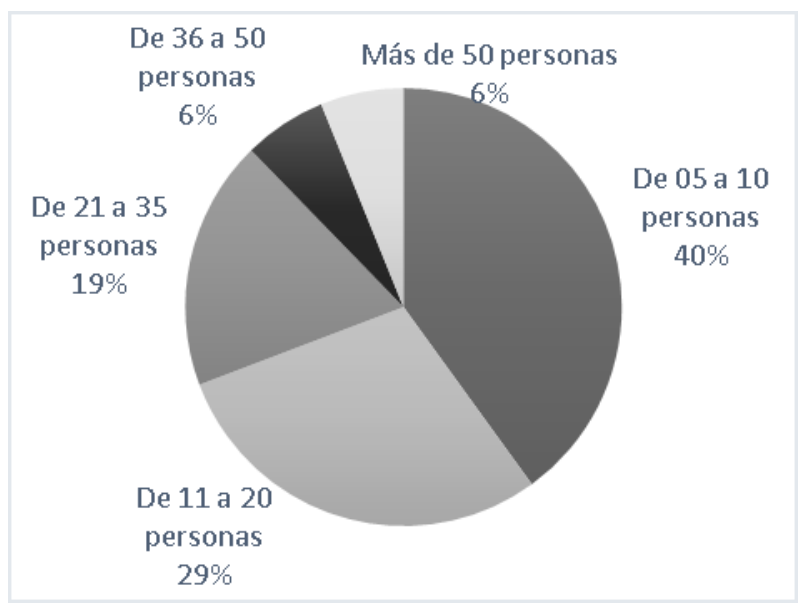

Figura 5. Distribución en función de la cantidad de empleados.

Algunas de las preguntas permitieron relacionar la incorporación de innovación por parte de la empresa.

Para evaluar la incorporación de diversas fuentes de innovación que indican adaptación a las nuevas tendencias de mercado y aplicación de las tecnologías, se indago sobre el uso de normas de calidad, uso de sistemas de colaboración en línea, TICs, nuevas inversiones en tecnologías para el negocio, uso de mecanismos de evaluación de satisfacción por parte del cliente, procesos de mejora continua para la prestación del servicio.

De estos datos se puede analizar que más del 70\% declaro haber incorporado Tecnologías de información y Comunicación en su negocio en los últimos 5 años, Figura 6, lo que indica la preponderancia que han tomado las herramientas informáticas en la administración correcta de un negocio.

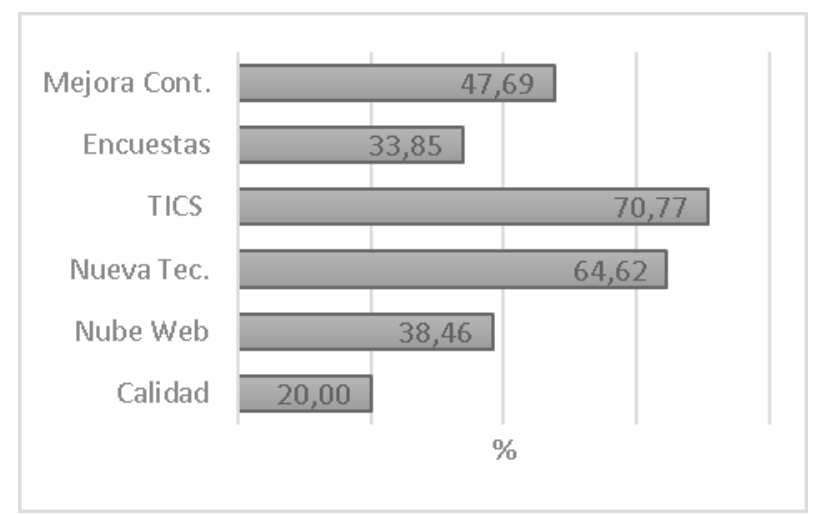

Figura 6. Incorporación de Innovación en la Organización 
El 64\% declara haber realizado alguna incorporación tecnológica para su negocio, de diversas índoles, pero asociadas con procesos de optimización y mejora del servicio o producto, indicando que la tecnología gana terreno continuamente y resulta recomendable o incluso necesaria su incorporación en algunos casos.

El 48\% declara ejecutar procesos de mejora continua sostenidos en el tiempo, lo cual demuestra el interés en innovar sobre la calidad y la prestación del servicio.

El 38\% declara utilizar tecnologías de colaboración en línea, lo que es un fuerte indicio de innovación en los tiempos de respuesta, siendo este tipo de herramientas, pensadas para agilizar el flujo de información y por tanto brindar un servicio más ágil.

El 33\% declara utilizar encuestas de satisfacción al cliente y solo el $20 \%$ reconoce el uso de normas y estándares de calidad en sus productos o servicios, lo que indica un interés menor por esta temática asociada a la calidad.

Puede inferirse de este análisis un mayor interés, hacia la mejora del servicio y su prestación, sesgada hacia la mejora del proceso, pero no hacia la opinión del cliente o a mantener estándares de calidad normalizados.

Se realizó otro análisis relacionado con el uso de las tecnologías de información y comunicación, enfocada a la publicidad. Figura 7.

Ello incluyo evaluar respuestas sobre el uso del correo electrónico y aplicaciones en dispositivos móviles, nubes, servidores de archivos, páginas web, medios de difusión masivos de correo electrónico y redes sociales.

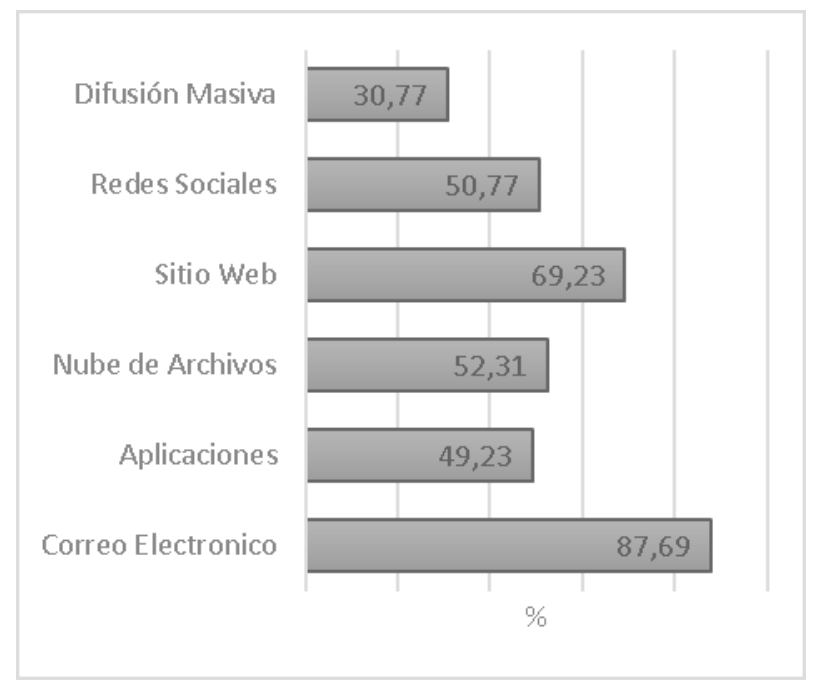

Figura 7. Utilización de Recursos Informáticos

El 87\% de los encuestados declaro utilizar el correo electrónico en los dispositivos móviles.

El 49\% declaro utilizar aplicaciones relacionadas para el desarrollo de sus tareas 
o coordinación, ello indica un uso intensivo de los dispositivos móviles y de las TICs para fortalecer las labores diariamente.

De igual manera el 52\% declara utilizar nubes de archivos para agilizar y sincronizar los trabajos dentro de su organización

El 70\% de los empresarios aseguraron tener un sitio WEB constituido y de uso permanente, lo que indica un elevado grado de interés por participar activamente en internet, posiblemente vinculado con la presencia de la empresa en el medio.

En relación a ello también es destacable que le 51\% admite utilizar las redes sociales para promocionar su empresa y productos, demuestra en concordancia un fuerte interés por parte de las PyMEs en este tipo de difusión y presencia para su organización.

No obstante es mencionable el hecho de que solo el 30\% de ellos envía difusión por mail, indicando posiblemente que este tipo de promoción no resulta de alto impacto, prefiriendo los otros medios citados.

Todo ello indica una fuerte adaptación por parte de los empresarios PyMEs a las nuevas tecnologías y al uso intensivo de estas y sus recursos tanto para mejorar la productividad como para promocionar la organización.

Para Evaluar la adaptación de estas tecnologías resulto de mucho interés conocer la longevidad con que estas empresas utilizan estas herramientas TICs.

Para ello se utilizaron preguntas relacionadas, segregado en diversos rangos característicos para realizar un análisis cuantitativo. Figura 8

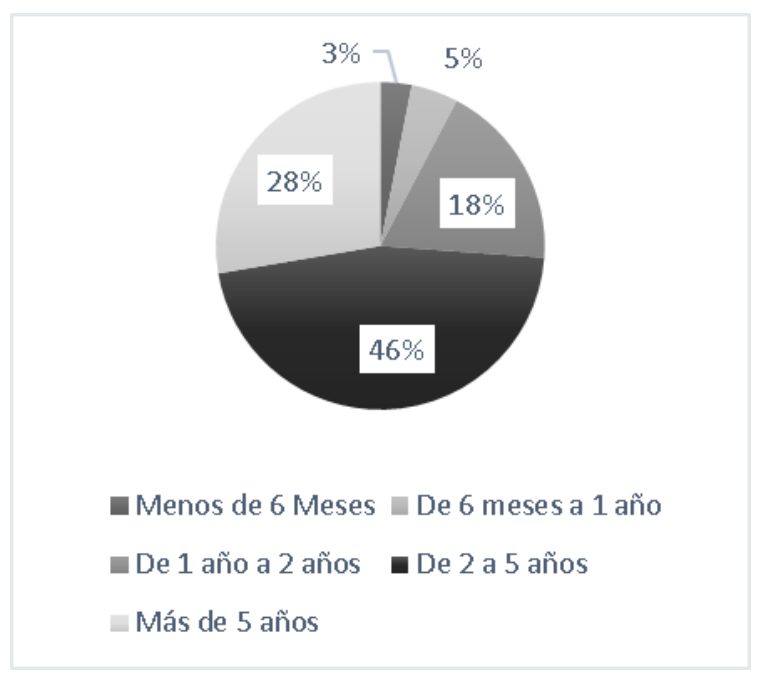

Figura 8. Tiempo transcurrido desde la implementación de los recursos informáticos

El 3\% de los encuestados informo utilizar los recursos informáticos en un periodo menor a 6 meses, un 5\% hasta 1 año, 18\% hasta 2 años, $46 \%$ hasta 5 años y $28 \%$ mayor a ese plazo.

Esto indica que las PyMes han innovado en el uso de estos recursos 
mayoritariamente en el último lustro.

Es importante recordar que el promedio de vida de las PyMEs encuestadas fue de 16 años.

\section{CONCLUSIONES}

La ejecución del proyecto permitió obtener una muestra representativa y correctamente distribuida de las PyMEs regionales.

El promedio de vida de estas empresas demuestra un elevado grado de madurez en general.

La porción mayoritaria de las empresas presenta un nivel total de empleo en el rango de 5 a 20 empleados.

La adaptación de estas empresas a las Tecnologías de Información y Comunicación es muy amplia, y los procesos asociados de incorporación de estas innovaciones han sido adoptados por la gran mayoría.

Las PyMEs no demuestran un interés prioritario en abocar sus esfuerzos a la normalización a partir de estándares.

Las PyMEs regionales han adaptado mayoritariamente los sistemas de correo electrónico y creación de sitios web, para mejorar la productividad de sus organizaciones.

Además la mitad de las organizaciones encuestadas declararon utilizar sistemas de Nubes de Archivos y Aplicaciones para dispositivos móviles para aumentar la productividad.

El 50\% de las organizaciones consultadas, dieron cuenta del uso de plataformas de Redes Sociales, como un factor relevante en términos publicitarios.

Mayoritariamente las empresas han incorporado estos recursos de las Tecnologías de información y Comunicación, en el transcurso de los últimos 5 años, por lo que se puede considerar reciente este tiempo de innovación en la región.

Se evidencia la adaptación de las PyMEs a las innovaciones tanto por las necesidades propias del mercado, como por la búsqueda de mejoras en la productividad.

\section{AGRADECIMIENTOS}

Este grupo de investigación agradece a todas las PyMEs que decidieron participar y colaborar en este proyectos, nuestro es el reconocimiento a todas ellas. 


\section{REFERENCIAS}

Bohlander G. y Snel S., (2006), "Managing human resources”. 6ta Edición, Cengage learnin, USA, 153-205.

Cuesta Santos A y Figueroa González J.M., , (2000)“Gestión estratégica de los recursos humanos”, 1ra Ed, Funiber, España, 28-29

Cuesta Santos A., , (2002)“Gestión del conocimiento. Análisis y proyección de los recursos humanos”. 1ra Ed. Academia, La Habana

Ferris G. R., Hall A. T., Royle M. T., y Martocchio J., (2004) “Theoretical development in the field of human resources Management" Organizational Analysis, Vol. 12, Issue 3, 231-254.

Llanes W., , (2004)“La dirección estratégica en la empresa” Ediciones centro de estudios de técnicas de dirección (CETDIR), Cuba.

Porter M. E.,(1982) "Estrategia competitiva: técnicas para el análisis de los sectores industriales y de la competencia” Editorial continental, Chile. Yoguel G.

Boscherini F., (1996) "La capacidad innovativa y el fortalecimiento de la competitividad de las firmas: el caso de las pymes exportadoras argentinas" CEPAL, Buenos Aires,

Harper y Lynch. , (1992)“Manuales de recursos humanos”, 1er Ed., Editorial Gaceta de negocios, Madrid.

Pena R. P. M., (2004) "Ética y estrategia en un marco teórico referencial de la ética de negocios” Revista de Administração Contemporânea, Vol 8, 229-252.

Mintzberg H., (2003) “The strategy process: concepts, contexts, cases”, 1ed, Pearson education, USA.

Gárciga R., (1999). "Formulación estratégica. Un enfoque para directivos", 1er Ed. Ediciones Félix Varela, Cuba.

Cuesta Santos A., (2005)“Tecnología de gestión de recursos humanos” 2da Ed. Academia, La Habana.

Sánchez Ortiz D. L., (2013)“Influencia de la calidad de vida laboral en los individuos y las organizaciones” Universidad de la sabana.

Hernandez Valencia R. A , (2009)“Estudio sobre los usos de las TIC en las Pymes del Área Metropolitana Centro Occidente de Colombia”, Revista de la Universidad Cooperativa de Colombia, Vol. 5 (12).

Castells, M., (2005) “La era de la información Vol.1. La sociedad red”, 1ed, Madrid, Alianza Editorial.

Vilaseca J., Torrent, J., (2003)“Las TIC y las transformaciones de la empresa catalana” 1ed, Universidad Oberta de Cataluña, España.

Peirano F., Suárez D.,(2006)“Tics y empresas: propuestas conceptuales para la generación de indicadores para la sociedad de la información”, Journal of Information Systems and Technology Management, Vol. 3 (2), 132-142. 